schließlich als Zusatztherapeutikum im Rahmen einer psychosozial betreuten Abstinenzbehandlung" hingewiesen wird. Die Verordnungen von Acamprosat sind seit Jahren rückläufig. Das Verordnungsvolumen kann nur einen minimalen Bruchteil der Alkoholkranken adäquat versorgen. Acamprosat ist grundsätzlich über einen Zeitraum von mindestens einem Jahr zu verordnen. Geht man mit der Deutschen Hauptstelle gegen die Suchtgefahren (DHS) von 1,6 Mio. Alkoholkranken in Deutschland aus, so reicht das Verordnungsvolumen gerade aus, um nominal 0,5\% der Kranken zu behandeln. Diese theoretische Bedarfsdeckung von nur 0,5\% ist vermutlich zu pessimistisch, indem vermutlich $80 \%$ der Alkoholkranken für diese Therapie nicht erreichbar sind. Unter dieser Prämisse wäre der Bedarf aber immer noch nur zu weniger als $3 \%$ gedeckt.

Dieser Unterversorgung will der Arzneiverordnungsreport anscheinend durch negative Suggestionen Vorschub leisten: Von den 17 publizierten, doppelblinden, plazebokontrollierten Studien mit insgesamt über 4500 Patienten zitiert der Arzneiverordnungsreport nur zwei, von denen eine keine Überlegenheit gegenüber Plazebo nachweisen konnte. Tatsächlich aber war Acamprosat in 15 der 17 Studien dem Plazebo signifikant überlegen, mit einer Effektstärke von rund 50\%. Die negative englische Studie erklärt sich vermutlich daraus, dass hier die Kranken nicht unmittelbar nach Entgiftung, sondern erst acht Wochen später rekrutiert wurden. Die deutsche Studie untersuchte auch ein Jahr nach Ende der Behandlung: Die Abstinenzrate war nach einem Jahr bei den zuvor mit Acamprosat behandelten Kranken mit 40\% immer noch signifikant höher als bei den mit Plazebo (17\%) behandelten.

\section{Korrespondenzadresse \\ Prof. Dr. med. Jürgen Fritze \\ Asternweg 65 \\ 50259 Pulheim}

Quelle: Schwabe U, Paffrath D (Hrsg): Arzneiverordnungsreport 2002. Springer-Verlag Berlin-Heidelberg 2003, ISBN 3-54043624-3

\title{
Die Nullrunde und die Psychiatrie
}

\author{
Jürgen Fritze \& Max Schmauß \\ für den Vorstand der Deutschen Gesellschaft für Psychiatrie, Psychotherapie und \\ Nervenheilkunde (DGPPN)
}

$\mathrm{D}$ as Gesetz zur Sicherung der Beitragssätze in der gesetzlichen Krankenversicherung und in der gesetzlichen Rentenversicherung hat u.a. die Nullrunde für die ambulante und stationäre Versorgung beschert, soweit die Krankenhäuser nicht für das neue DRGEntgeltsystem optiert haben. Von dieser Option haben bis 31.10.2002 530 Krankenhäuser Gebrauch gemacht. Unter dem Druck der Deutschen Krankenhausgesellschaft (DKG) hat das BMGS das 12. SGB V Änderungsgesetz dahingehend ergänzt, dass die Frist zum Optieren über den ursprünglich geltenden Termin 31.10.2002 hinaus bis zum 31.12.2002 verlängert würde. Das Gesetz ist bisher am Widerstand des Bundesrates gescheitert. Es ist aber zu erwarten, dass man sich zumindest bezüglich der verlängerten $\mathrm{Op}$ tionsfrist doch einigen wird.

Psychiatrische und psychosomatische Einrichtungen sind vom DRGSystem gemäß § 17b KHG ausgenommen, können also nicht optieren, werden also von der Nullrunde getroffen. Mindestens 20\% der somatisch Kranken weisen behandlungsbedürftige psychiatrische Komorbiditäten auf. Um eigene Ressourcen zu schonen, werden die optierenden somatischen Häuser diese Komorbiditäten dazu nutzen, diese Kranken frühzeitig in psychiatrische und psychosomatische Einrichtungen zu verlegen.

Nachdem bereits rund ein Viertel der Krankenhäuser bekundet hat, von der Option Gebrauch zu machen, und die verlängerte $\mathrm{Er}$ klärungsfrist diesen Anteil auf 60\% vergrößern wird, ist zu erwarten, dass im Jahr 2003 psychiatrische und psychosomatische Einrichtungen mit einer erheblichen Fallzahlsteigerung konfrontiert sein werden. Dem können sie bei gesetzlich ver- ordneter Nullrunde, d.h. ohne zusätzliche Ressourcen, nicht gewachsen sein. Hierauf wurde das BMGS im Vorfeld des Gesetzgebungsverfahrens hingewiesen, sah aber keinen Handlungsbedarf.

Dabei ist es fragwürdig, ob die Chancenlosigkeit psychiatrischer Einrichtungen, der Nullrunde zu entgehen, überhaupt verfassungskonform ist, denn sie verstößt gegen das Gleichheitsgebot des Grundgesetzes. Sie hebelt darüber hinaus geltendes Recht aus: Die Bundespflegesatzverordnung (BPflV) sieht ausdrücklich vor, dass der Gesamtbetrag (das Budget) des Vorjahres überschritten werden darf, wenn die Vorgaben der Psychiatrie-Personalverordnung zur Zahl der Personalstellen auf der Basis der Stichtagserhebungen dies erforderlich machen. Dabei stellt der Verordnungsgeber ausdrücklich fest, dass sicherzustellen ist, dass das Personal nicht anderweitig eingesetzt wird.

Dies hat der Ausschuss für Gesundheit und Soziale Sicherung des Bundestages am 13.11.2002 ausdrücklich herausgestellt: „Von den Vertretern der Koalitionsfraktionen wurde ausdrücklich darauf hingewiesen, dass für die Psychiatrischen Krankenhäuser die Psychiatrie-Personalverordnung uneingeschränkt gelte. Psychiatrische Krankenhäuser hätten somit unabhängig von der Grundlohnrate Anspruch auf die Refinanzierung einer der PsychiatriePersonalverordnung entsprechenden Personalausstattung. Zusätzlich hätten die Psychiatrischen Krankenhäuser Anspruch auf die übrigen Ausnahmetatbestände des $\S 6$ Abs. 1 BPfIV und somit auch Anspruch auf BAT-Ausgleich“.

\section{Korrespondenzadresse \\ Prof. Dr. med. Jürgen Fritze \\ Asternweg 65}

50259 Pulheim 